\title{
The OTELO Survey: The Star Formation Rate Evolution of Low-mass Galaxies
}

\author{
Bernabé Cedrés ${ }^{1,2}$ (1) , Ana María Pérez-García ${ }^{3,4}$, Ricardo Pérez-Martínez ${ }^{4,5}$, Miguel Cerviño ${ }^{3}$, Jesús Gallego ${ }^{6}$ (1), \\ Ángel Bongiovanni ${ }^{4,7}$, Jordi Cepa ${ }^{1,2,4}$, Rocío Navarro Martínez ${ }^{4}$, Jakub Nadolny ${ }^{1,2}$ (D), Maritza A. Lara-López ${ }^{8}$ (D), \\ Miguel Sánchez-Portal $^{4,7}$, Emilio J. Alfaro ${ }^{9}$, José A. de Diego ${ }^{10}$, Mauro González-Otero ${ }^{1,2,4}$, J. Jesús González ${ }^{10}$, \\ J. Ignacio González-Serrano ${ }^{11}$, and Carmen P. Padilla Torres ${ }^{4,12,13}$ \\ ${ }^{1}$ Instituto de Astrofísica de Canarias (IAC), E-38200 La Laguna, Tenerife, Spain; bcedres@iac.es \\ 2 Departamento de Astrofísica, Universidad de La Laguna (ULL), E-38205 La Laguna, Tenerife, Spain \\ ${ }^{3}$ Centro de Astrobiología (CSIC/INTA), E-28692 ESAC Campus, Villanueva de la Cañada, Madrid, Spain \\ ${ }^{4}$ Asociación Astrofísica para la Promoción de la Investigación, Instrumentación y su Desarrollo, ASPID, E-38205 La Laguna, Tenerife, Spain \\ 5 ISDEFE for European Space Astronomy Centre (ESAC)/ESA, P.O. Box 78, E-28690 Villanueva de la Cañada, Madrid, Spain \\ ${ }^{6}$ Departamento de Física de la Tierra y Astrofísica, Instituto de Física de Partículas y del Cosmos, IPARCOS. Universidad Complutense de Madrid, E-28040, Madrid, \\ Spain \\ ${ }^{7}$ Institut de Radioastronomie Millimétrique (IRAM), Av. Divina Pastora 7, Núcleo Central E-18012, Granada, Spain \\ ${ }^{8}$ Armagh Observatory and Planetarium, College Hill, Armagh BT61 9DG, Northern Ireland, UK \\ ${ }_{9}$ Instituto de Astrofísica de Andalucía, CSIC, E-18080, Granada, Spain \\ ${ }^{10}$ Instituto de Astronomía, Universidad Nacional Autónoma de México, Apdo. Postal 70-264, 04510 Ciudad de México, Mexico \\ ${ }^{11}$ Instituto de Física de Cantabria (CSIC-Universidad de Cantabria), E-39005 Santander, Spain \\ ${ }^{12}$ INAF, Telescopio Nazionale Galileo, Apartado de Correos 565, E-38700 Santa Cruz de la Palma, Spain \\ ${ }^{13}$ Fundación Galileo Galilei-INAF Rambla José Ana Fernández Pérez, 7, E-38712 Breña Baja, Tenerife, Spain \\ Received 2021 April 13; revised 2021 June 11; accepted 2021 June 11; published 2021 July 1
}

\begin{abstract}
We present the analysis of a sample of $\mathrm{H} \alpha, \mathrm{H} \beta$, and [O II] emission line galaxies from the OTELO survey, with masses typically below $\log \left(M_{*} / M_{\odot}\right) \sim 9.4$ and redshifts between $z \sim 0.4$ and 1.43 . We study the star formation rate, star formation rate density, and their number density and evolution with redshift. We obtain a robust estimate of the specific star formation rate-stellar mass relation based on the lowest-mass sample published so far. We also determine a flat trend of the star formation rate density (SFRD) and number density with redshift. Our results suggest a scenario of no evolution of the number density of galaxies, regardless of their masses, up to redshift $z \sim 1.4$. This implies a gradual change of the relative importance of the star-forming processes, from high-mass galaxies to low-mass galaxies, with decreasing redshift. We also find little or no variation of the SFRD in the redshift range of $0.4<z<1.43$.
\end{abstract}

Unified Astronomy Thesaurus concepts: Luminosity function (942); Observational cosmology (1146); Star formation (1569); Starburst galaxies (1570); Surveys (1671)

\section{Introduction}

The study of star formation along the cosmic times provides an outstanding insight into the main physical processes driving the evolution of galaxies at cosmological scales. There is a tight relationship between star formation rate (SFR) and stellar mass for star-forming galaxies-the main sequence (MS)—in both the local and high-redshift universe (see Brinchmann et al. 2004, and many others). Focusing on the low-mass range, the study of the SFR of dwarf galaxies allows us to establish a direct connection to the early epochs of galaxy formation. Whitaker et al. (2014) found that the evolution of low-mass galaxies is steeper than in massive galaxies for galaxies with masses larger than $10^{9} M_{\odot}$, using SFR obtained from ultraviolet (UV) and far-infrared (FIR) continuum. In the same way, González et al. (2014) have shown that for sources with $M_{*} \sim 5 \times 10^{9} M_{\odot}$ the specific star formation rate (sSFR) shows no evidence of significant evolution from $z \sim 2$ to $z \sim 7$ $\left(\mathrm{sSFR} \sim 2 \mathrm{Gyr}^{-1}\right)$. On the other hand, results of the EAGLE and Illustris simulations suggest that low-mass galaxies behave differently than more massive ones, agreeing with observations for masses larger than $10^{9} M_{\odot}$ (Schaye et al. 2015; Vogelsberger et al. 2014).

Previous works have shown that the star formation rate density (SFRD) declines along cosmic time (see, for example, Harish et al. 2020; Sobral et al. 2013), although the samples used are composed by high-mass galaxies. Some studies, such as Mobasher et al. (2009) who used a sample of galaxies with masses larger than $10^{9.5} M_{\odot}$, concluded that the massive systems have had their major star formation activity at earlier epochs $(z>2)$ than the lower-mass galaxies. Davies et al. (2009), analyzing a sample of dwarf galaxies at $z \sim 1$, suggested that for low-mass galaxies, the SFRD is roughly constant from $z=1$ to now. However, there are no conclusive studies for low-mass galaxies at non-local redshifts, mainly because of the difficulty of detecting such faint objects.

In this Letter, we present the results of the analysis of the star formation activity of a sample of galaxies with masses as low as $\log \left(M_{*} / M_{\odot}\right) \sim 7.12$, with $80 \%$ of them below $\log \left(M_{*} / M_{\odot}\right) \sim 9.4$ at redshifts $\sim 0.4-1.4$ from the OTELO survey, with the purpose of establishing the evolution of the SFR and SFRD for these low-mass objects.

Throughout this Letter we assume a standard $\Lambda$-cold dark matter cosmology with $\Omega_{\Lambda}=0.7, \quad \Omega_{m}=0.3, \quad$ and $\mathrm{H}_{0}=70 \mathrm{~km} \mathrm{~s}^{-1} \mathrm{Mpc}^{-1}$.

\section{The OTELO Samples}

This work takes advantage of the OTELO survey and its data products (Bongiovanni et al. 2019). The OTELO survey is a narrow-band-scan ultra-deep pencil-beam survey carried out with tunable filters of the OSIRIS instrument on the Gran 
Table 1

Data Sample Description and Luminosity Functions

\begin{tabular}{lccccr}
\hline \hline Employed Emission Line & $\begin{array}{c}\text { Number of } \\
\text { Emitters }\end{array}$ & $\langle z\rangle$ & $\begin{array}{c}\left\langle V_{c}\right\rangle \\
\left(10^{3} \mathrm{Mpc}^{3}\right)\end{array}$ & $\begin{array}{c}\log \phi^{*} \\
\left(\mathrm{Mpc}^{-3} \mathrm{dex}^{-1}\right)\end{array}$ & $\begin{array}{c}\log L^{*} \\
\left(\mathrm{erg} \mathrm{s}^{-1}\right)\end{array}$ \\
\hline H $\alpha$ (Ramón-Pérez et al. 2019) & 46 & 0.40 & 1.40 & $-2.75 \pm 0.19$ & $41.85 \pm 0.22$ \\
H $\beta$ (Navarro Martínez et al. 2021) & 40 & 0.90 & 5.19 & $-2.77 \pm 0.12$ & $41.34 \pm 0.27$ \\
[O II] (Cedrés et al. 2021) & 60 & 1.43 & 10.21 & $-3.23 \pm 0.11$ & $42.44 \pm 0.11$ \\
\hline
\end{tabular}

Telescopio Canarias. It covers a region of $56 \operatorname{arcmin}^{2}$ of the Extended Groth Field. Observations through 36 tunable filter images in the 8950-9300 A region provides a pseudospectra $(\mathrm{R} \sim 700)$ from which emission lines can be identified and measured. The limiting flux detected in the OTELO survey is $\sim 1.10^{-19}$ erg $\mathrm{cm}^{-2} \mathrm{~s}^{-1} \AA^{-1}$, which constitutes the deepest narrow-band survey to date. These data are complemented with $u, g, r, i, z, J, H$, and $K_{s}$ photometry. Redshift and extinction estimates has been obtained from the Le Phare code (Ilbert et al. 2006) using representative templates of Hubble types from Coleman et al. (1980), and starburst galaxies from Kinney et al. (1996) and different $E(B-\mathrm{V})$ assuming Calzetti et al. (2000) law. The estimations of $E(B-V)$ values were sampled by the code in intervals of $0.05 \mathrm{mag}$. We obtained median extinctions $\quad E(B-V) \simeq 0.1, \quad E(B-V) \simeq 0.1, \quad$ and $E$ $(B-V) \simeq 0.15$ for $\mathrm{H} \alpha, \mathrm{H} \beta$, and [O II] respectively (see Ramón-Pérez et al. 2019; Navarro Martínez et al. 2021 and Cedrés et al. 2021).

A direct consequence of the design of the OTELO survey is the favoring of detection of low-mass galaxies $\left(M_{*}<10^{10} M_{\odot}\right)$, which comprise more than the $87 \%$ of the total detected emitters (see, for example, Cedrés et al. 2021 and Bongiovanni et al. 2020).

The luminosity functions (LFs) covering the main emission lines for the OTELO emitters has already been calculated in Ramón-Pérez et al. (2019), Navarro Martínez et al. (2021), and Cedrés et al. (2021) for redshifts $z \sim 0.4(\mathrm{H} \alpha), z \sim 0.9(\mathrm{H} \beta)$ and $z \sim 1.43$ ([O II]), respectively. An in-depth description of the methods and corrections applied (including the corrections from the presence, completeness, and cosmic variance of active galactic nuclei (AGNs)) can be found in those works.

In Table 1, a summary of the data employed in this study is presented. This table includes the number of emitters, the volume sampled at each band observed, and the Schechter (1976) parameters for the LF fit.

\section{SFR and SSFR}

The SFR was calculated following Kennicutt (1998), employing a Kroupa (2001) initial mass function (IMF).

$$
\begin{gathered}
\operatorname{SFR}\left(M_{\odot} \mathrm{yr}^{-1}\right)=5.29 \times 10^{-42} L(\mathrm{H} \alpha)\left(\mathrm{erg} \mathrm{s}^{-1}\right), \\
\operatorname{SFR}\left(M_{\odot} \mathrm{yr}^{-1}\right)=1.507 \times 10^{-41} L(\mathrm{H} \beta)\left(\mathrm{erg} \mathrm{s}^{-1}\right),
\end{gathered}
$$

for $\mathrm{H} \alpha$ and $\mathrm{H} \beta$, respectively. For [O II], due to its dependence on metallicity, we choose to use the improved Kennicutt (1998) calibration proposed by Kewley et al. (2004),

$$
\operatorname{SFR}\left(M_{\odot} \mathrm{yr}^{-1}\right)=\frac{5.29 \times 10^{-42} L([\mathrm{O} \mathrm{II}])\left(\mathrm{erg} \mathrm{s}^{-1}\right)}{-1.75 \times[\log (\mathrm{O} / \mathrm{H})+12]+16.73},
$$

where $\log (\mathrm{O} / \mathrm{H})+12$ is the oxygen abundance for each of the [O II] emitters. Based on Henry et al. (2013), we assumed a mean value for the metallicity for the gas phase of dwarf galaxies at our redshift of $\sim 8.5$. To derive the uncertainties due to the use of this mean value of the metallicity instead of a properly derived quantity for each emitter, we assumed that the real abundance could vary from a minimum of $\sim 8.2$ to a maximum of $\sim 8.8$ (values given in Henry et al. 2013). Then, we calculated the values for the SFR for the minimum and maximum metallicity and used them as extreme values for the SFR. The differences between the extreme SFRs and the SFR from the mean oxygen abundance gives us the uncertainty range in the SFR due to the metallicity dependence.

The Le Phare code (see Section 2) does not provide an evaluation of the errors in $E(B-V)$, therefore the uncertainty in this term was not taken into account when determining the uncertainty in the SFR. Nevertheless, the derived values of $E$ $(B-V)$ are small, therefore the effect of dust extinction on the derived SFRs should be small, although it would introduce some extra scatter in our SFR estimates. It should also be noted that by taking into account the fact that all of the lines employed in this study sample a similar region of the ionizing continuum, and hence the same timescale of the star formation activity (see, for example, Cerviño et al. 2016 and references therein), we can assume that all SFRs derived can be compared with each other.

The stellar mass for each emitter was derived following López-Sanjuan et al. (2019) for quiescent and SFGs, employing rest-frame $g$ - and $i$-band magnitudes, together with absolute $i$-band magnitude (see Nadolny et al. 2020 for details). The range of the masses derived were $10^{7.1}<M_{*} / M_{\odot}<10^{9.6}$ for $\mathrm{H} \alpha \quad 10^{7.6}<M_{*} / M_{\odot}<10^{10.7}$ for $\mathrm{H} \beta$ and $10^{7.89}<M_{*} / M_{\odot}<10^{10.93}$ for [O II].

In Figure 1 panel (a) we have represented the SFR as a function of the stellar mass of the galaxies. A small difference in the SFR with redshift may exist, with the $\mathrm{H} \alpha$-detected galaxies having lower values of the SFR when compared with the galaxies detected in $\mathrm{H} \beta$ and [O II]. This difference may be attributed to a selection effect of the sources. Indeed, the mean value of the $\mathrm{SFR}$ in $\mathrm{H} \alpha$ is $0.125 M_{\odot} \mathrm{yr}^{-1}$, so if we want to observe such SFR in the [O II] line, the value of the luminosity has to be $2.36 \times 10^{40} \mathrm{erg} \mathrm{s}^{-1}$. However, the minimum value in luminosity detected for the [O II] galaxy sample is $2.37 \times 10^{40} \mathrm{erg} \mathrm{s}^{-1}$ (see Cedrés et al. 2021). Nevertheless, taking into account the uncertainties, the results for $\mathrm{H} \alpha$ are compatible with the rest of the lines ( $\mathrm{H} \beta$ and [O II] $)$ in the mass range in which they coincide.

In Figure 1, panel (b) we have represented the logarithm of the sSFR as a function of the logarithm of the stellar mass, stacked in bins of $0.5 \mathrm{dex}$ in $\log \left(M_{*}\right)\left[M_{\odot}\right]$. It seems that the results for the [O II] emitters present a larger value for the sSFR when compared with the $\mathrm{H} \alpha$ and $\mathrm{H} \beta$ emitters; however, if we take into account the uncertainties, this figure suggests that the bulk of all the emitters, regardless of the observed line, are located inside the area of the MS defined by the number of galaxies from the SDSS database (Renzini \& Peng 2015). 


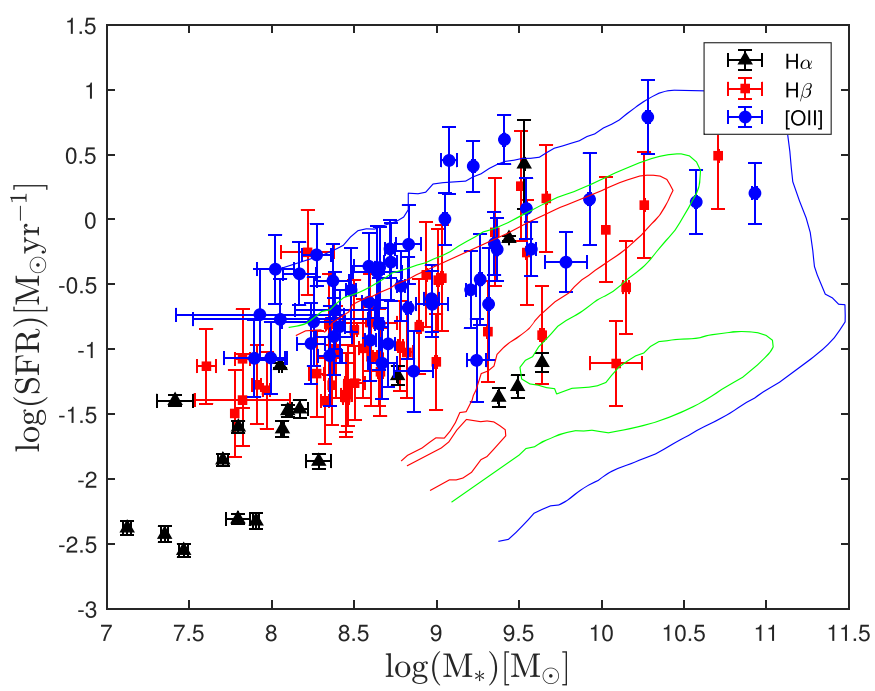

(a)

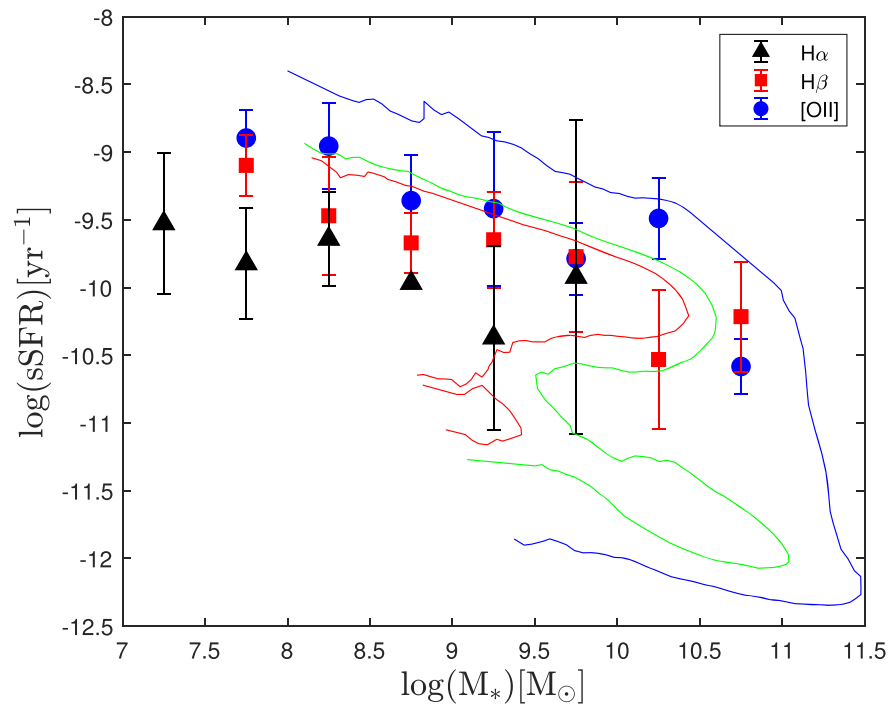

(b)

Figure 1. Logarithm of the SFR (panel a) and the stacked sSFR (panel b) as a function of the logarithm of the stellar mass. Black triangles are the data from H $\alpha$ (Ramón-Pérez et al. 2019), red squares are the data from H $\beta$ (Navarro Martínez et al. 2021), and blue circles are the data from [O II] emitters (Cedrés et al. 2021). Contours correspond to the number of galaxies from the Sloan Digital Sky Survey (SDSS) database obtained by Renzini $\&$ Peng (2015) at values of $2.0 \times 10^{4}$ (blue), $7.0 \times 10^{4}$ (green), and $1.2 \times 10^{5}$ (red), the last one being the definition of the local star-forming MS.

Following Gilbank et al. (2011), we may assume a powerlaw relationship between the sSFR and the stellar mass, such as sSFR $\propto M_{*}^{\beta}$. Our data sample have masses down to $\log \left(M_{*} / M_{\odot}\right) \sim 7.12$ for $\mathrm{H} \alpha$, and median values of $\log \left(M_{*} / M_{\odot}\right)=8.06, \quad \log \left(M_{*} / M_{\odot}\right)=8.62 \quad$ and $\log \left(M_{*} / M_{\odot}\right)=8.72$, with $80 \%$ of galaxies below the masses $\log \left(M_{*} / M_{\odot}\right)=9.4, \quad \log \left(M_{*} / M_{\odot}\right)=9.5 \quad$ and $\log \left(M_{*} / M_{\odot}\right)=9.3$ for $\mathrm{H} \alpha, \mathrm{H} \beta$ and [O II] respectively. We obtain a least-squared fit to these stacked data with $\beta_{\mathrm{H} \alpha}=-0.1 \pm 0.2, \beta_{\mathrm{H} \beta}=-0.5 \pm 0.2$ and $\beta_{[\mathrm{OIII}]}=-0.6 \pm 0.3$.

This is consistent with previous works performed with higher-mass ranges. Ramraj et al. (2017) obtained the sSFR for the $\mathrm{H} \alpha$ line at $z \sim 1$ in the mass range $8.5<\log \left(M_{*} / M_{\odot}\right)<9.5$, and a value of $\beta=-0.47 \pm 0.04$, and also from the data obtained by Gilbank et al. (2010), a value of $\beta=-0.08 \pm 0.01$ for $z \sim 0.1$ in that mass range.

The depth of the data used is crucial to determine the slope of the sSFR-stellar mass relation, as also noted in Ramraj et al. (2017). The OTELO survey is able to sample the lowest-mass ranges published to date for the objects that we study in this Letter, allowing us to provide the most robust estimation of the steepness of this relation. Our results reinforce the idea of flatter slopes for $\beta$ at intermediate redshifts than the previous ones presented in literature (see, for example, Sobral et al. 2011, where they obtained $\beta=-1.0 \pm 0.07$ at $z \sim 1$ ).

\section{SFR Density and Number Density Evolution}

\subsection{SFR Density}

As explained in Section 2, the LF has been obtained for each band employed in this study. This LF may be integrated to obtain the total luminosity density. According with Schechter (1976), the integral is equal to

$$
\mathcal{L}=\int_{0}^{\infty} \phi(L) L d L=\phi^{\star} L^{\star} \Gamma(\alpha+2),
$$

where $\Gamma$ is the gamma function.
This integrated luminosity density can be converted into SFRD by simply substituting the normal luminosity $L$ by $\mathcal{L}$ in Equations (1), (2) and (3) for $\mathrm{H} \alpha, \mathrm{H} \beta$, and [O II] respectively.

In Figure 2 panel (a) we have represented the SFRD as a function of the redshift. Our data are the black dots $(\mathrm{H} \alpha, \mathrm{H} \beta$, and [O II] $)$, the open cyan circles are data from Ly et al. (2007), Morioka et al. (2008), Harish et al. (2020), and Sobral et al. (2013), all derived from the integration of their proposed LFs. The red star is the data for dwarf galaxies only from Davies et al. (2009). It is clear from the OTELO data that there is little to no evolution of the SFRD for low-mass galaxies. Moreover, OTELO results seem to be closer to the one obtained by Davies et al. (2009). Cedrés et al. (2021) discussed how part of the low value for the SFRD in [O II] may be explained by the large uncertainty presented in $\phi^{*}$ due to the cosmic variance (CV). The same may happen with the other lines. Nevertheless, this effect may include uncertainty on $\phi^{*}$ of about $100 \%$, which has been taken into account when deriving the errorbars in the SFRD. Moreover, the uncertainty due to the $\mathrm{CV}$ will have a random effect in the final value of the SFRD, but in our case, all the lines present the same behavior, which makes it compatible with a no-evolution scenario.

On the other hand, there is an increase in the SFRD with redshift for samples containing low-mass and high-mass galaxies. For low redshift, there is an agreement between OTELO and the data from literature (Ly et al. 2007 for emitters at $z \sim 0.07$, Morioka et al. 2008 for emitters at $z \sim 0.24$ or Sobral et al. 2013 for emitters at $z \sim 0.4$ ). This confirms the suggestion of Davies et al. (2009), who pointed out that the bulk of the SFRD shifts from high-mass to low-mass galaxies with decreasing redshift. Meanwhile, the SFRD in low-mass galaxies has remained fairly constant with redshift, and it is a clear indication of the downsizing first suggested in Cowie et al. (1996) and detected in Weisz et al. (2011) or in RodríguezMuñoz et al. (2015), where it is hinted that at least $90 \%$ of the stellar mass for low-mass galaxies is preferably formed at low redshift $(z \lesssim 0.15)$. 


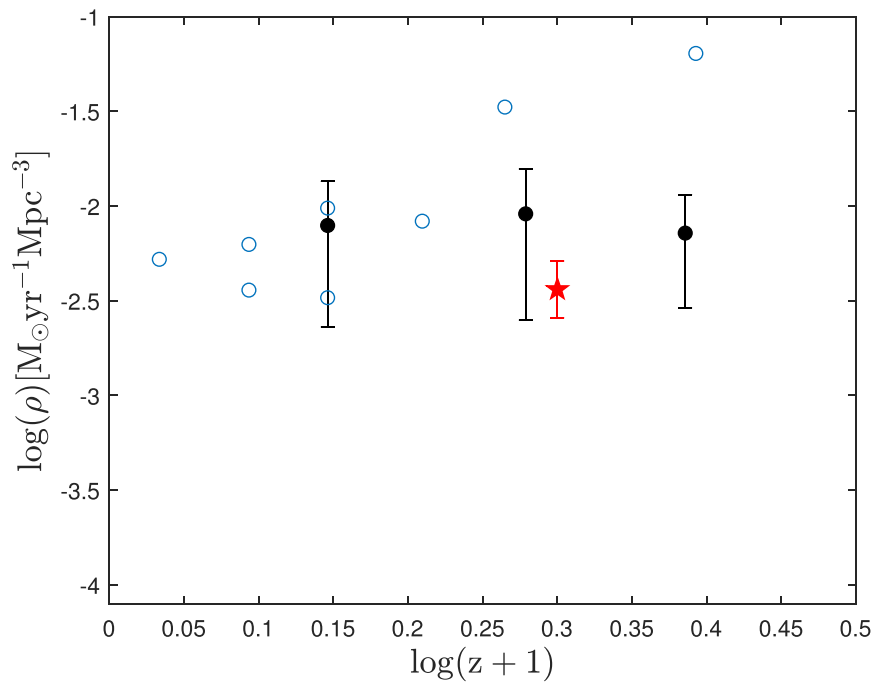

(a)

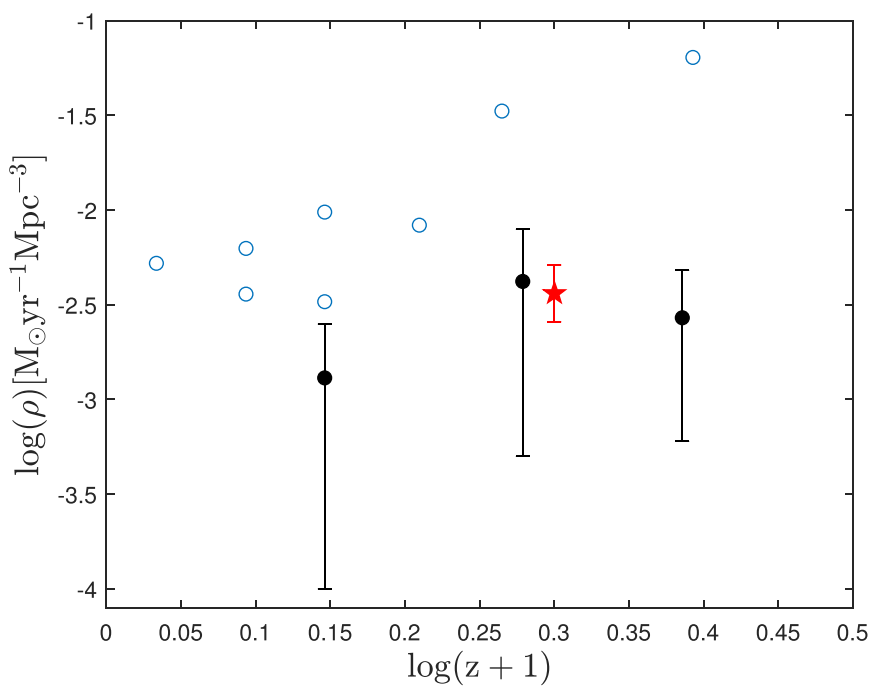

(b)

Figure 2. Star formation density as a function of $\log (z+1)$. Our results are the black dots. Blue circles are the data from Ly et al. (2007), Morioka et al. (2008), Harish et al. (2020), and Sobral et al. (2013); the red star is the result from only low-mass galaxies extracted from Davies et al. (2009). The SFRD for all the data is calculated employing Kroupa (2001) IMF. In panel (a) the integration is performed up to infinity in mass, in panel (b) it is performed up to $M_{*}<10^{9} M_{\odot}$.

In Figure 2 panel (b) we have represented the SFRD for OTELO survey integrated up to $M_{*}=10^{9} M_{\odot}$ (black dots), to include only low-mass galaxies. This was done in order to better compare our results with that from Davies et al. (2009) and represented as a red star. Indeed, the main body of our data comes from low-mass galaxies, but the integration of the LF from Equation (4) is performed up to infinity, which includes some contribution by high-mass galaxies that may bias the value of the derived SFRD.

We can obtain the contribution to the luminosity density of only the low-mass galaxies analytically by doing the following:

$$
\begin{aligned}
& \mathcal{L}_{\text {low }- \text { mass }}=\mathcal{L}_{\text {total }}-\mathcal{L}_{\text {high-mass }} \\
& \quad=\phi^{*} L^{*}\left[\Gamma(\alpha+2)-\Gamma\left(\alpha+2, L / L^{*}\right)\right],
\end{aligned}
$$

where $\Gamma\left(\alpha+2, L / L^{*}\right)$ is the incomplete gamma function, and $L$ is the maximum value for the luminosity to integrate the luminosity density, corresponding to the luminosity of the maximum value of the stellar mass, in this case $M_{*}=10^{9} M_{\odot}$. To estimate the limiting value of $L$, we assumed a linear relationship between the continuum of the emitters at the observed bands and the stellar mass, and obtained a limiting $L_{\text {cont }}$ for the emitters with $M_{*} \leqslant 10^{9} M_{\odot}$. We also assumed that the equivalent width of the lines is approximately constant for low masses up to $M_{*}=10^{9} M_{\odot}$. We know that this is a crude approximation, but the assumption of a constant equivalent width is roughly valid for our sample, as it is shown in Figure 9 of Cedrés et al. (2021) for the case of [O II] emitters. Employing this, we were able to determine, in a somewhat crude way, a limiting value for the luminosity of the line $L$ from Equation (5). The obtained limits were $\log \left(L\left[\operatorname{erg} \mathrm{s}^{-1}\right]\right)=40.26,40.71$, and 41.27 for $\mathrm{H} \alpha, \mathrm{H} \beta$, and [O II] respectively. With those values, the resulting $\mathcal{L}_{\text {low-mass }}$ from Equation (5) is then converted in SFRD following Equations (1), (2) and (3).

From Figure 2 panel (b), we can see that the agreement of our values of the SFRD with the results from Davies et al. (2009) is now clearer. However, it seems that the $\mathrm{H} \alpha$ emitters may present a lower value of the SFRD when compared with the other emitters, even if the general result is well inside the derived uncertainties. This can be explained by the very low sampled volume for the $\mathrm{H} \alpha$ emitters (see Table 1), which is 5 and 10 times smaller in this line than in $\mathrm{H} \beta$ and [O II], respectively. This implies a larger uncertainty due to the cosmic variance (Stroe \& Sobral 2015) when compared with $\mathrm{H} \beta$ and [O II]. Even so, it seems that the non-evolutionary scenario is preserved.

\subsection{Number Density}

Each LF can also be integrated to obtain the corresponding number density $(\mathcal{N})$ for each redshift sample $(\mathrm{H} \alpha, \mathrm{H} \beta$, and [O II]).

$$
\mathcal{N}=\int_{0}^{\infty} \phi(L) d L
$$

In this case, we employed a numerical integration technique. As integration limits, we used $38<\log (L)$ [erg/s] $<43$ to cover the full range of the possible masses of the emitters. We obtained $\log \left(\mathcal{N}_{\mathrm{H} \alpha}\left[\mathrm{Mpc}^{-3}\right]\right)=-1.34_{-0.53}^{+0.23}$, $\log \left(\mathcal{N}_{\mathrm{H} \beta}\left[\mathrm{Mpc}^{-3}\right]\right)=-0.99_{-0.40}^{+0.21}$ and $\log \left(\mathcal{N}_{[\mathrm{OII}]}\left[\mathrm{Mpc}^{-3}\right]\right)=-1.02_{-0.48}^{+0.22}$. In Figure 3 we have represented the number density as a function of the logarithm of the redshift for the $\mathrm{H} \alpha, \mathrm{H} \beta$, and [O II] bands in the OTELO survey (black dots). For comparison, we also have represented data from Ly et al. (2007), Drake et al. (2013), Khostovan et al. (2015), and Hayashi et al. (2013) (cyan dots).

The data points from previous works include galaxies not filtered by mass that are in general more massive than those in our sample. The scatter reflects a non-conclusive trend in the evolution with redshift, especially in the range of $\mathrm{z} \sim 0.8$. Our work, based mainly on low-mass galaxies $\left(M_{*}<10^{10} M_{\odot}\right)$, clearly establishes a flat slope in the evolution of the number density with redshift. 


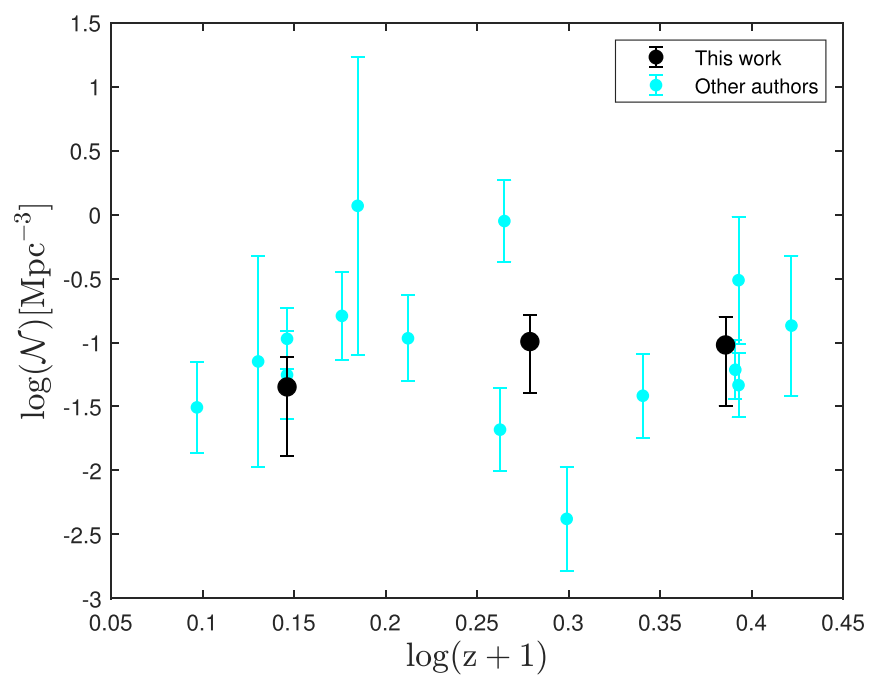

Figure 3. Number density as a function of redshift for the OTELO emitters (black dots), and authors from the literature (cyan dots). The figure includes data from Ly et al. (2007) (H $\alpha$, [O III] and [O II]), Drake et al. (2013) (H $\alpha$ and [O II]), Khostovan et al. (2015) (H $\beta$ ), and Hayashi et al. (2013) ([O II]).

\section{Discussion and Conclusions}

By estimating the SFR for low-mass galaxies in the three groups of emitters from $0.4<z<1.43$ detected in the OTELO survey, we find little or no evolution along with this redshift range. The different limiting magnitude achieved at each redshift window, with the lowest-mass galaxies detected at $z \sim 0.4$, explains the apparent lower values for the SFR and sSFR at this redshift. At the same time, the smaller cosmic volume mapped explains the lack of high-mass $\mathrm{H} \alpha$ emitters.

Nevertheless, the data for all the emitters follow the MS defined by Renzini \& Peng (2015) for both the SFR and sSFR.

We fitted a power law to the stacked and binned sSFR relation with the stellar mass, obtaining $\beta_{\mathrm{H} \alpha}=-0.1 \pm 0.2$, $\beta_{\mathrm{H} \beta}=-0.5 \pm 0.2$ and $\beta_{[\mathrm{OII}]}=-0.6 \pm 0.3$. These results expand previous works (Ramraj et al. 2017) to masses as low as $\log \left(M_{*} / M_{\odot}\right)=7.12$ and to redshifts up to 1.43 . We find shallower slopes when comparing with Sobral et al. (2011). This flatter slope in the relationship between sSFR versus the stellar mass indicates a more constant star formation history of low-mass galaxies, as suggested by Ramraj et al. (2017).

By integrating the LFs for each group, we were able to derive the SFRD and we represented it as a function of the redshift. We find no evolution with $z$ of the SFH for our sample, formed mainly by low-mass galaxies. Even more, when integrating only objects up to $M_{*}=10^{9} M_{\odot}$ we get the same result. The evolution found in previous works (Ly et al. 2007; Morioka et al. 2008; Sobral et al. 2013; Harish et al. 2020) would come from the contribution of higher-mass galaxies. The value we obtained with $\mathrm{H} \alpha$ data seems to be somewhat smaller than those from the other emitters. Nevertheless, its value is within the uncertainties. This may be attributed to the cosmic variance effect due to the smaller volume sampled when compared with the $\mathrm{H} \beta$ and [O II] data. These results confirm the ones obtained by Davies et al. (2009) for low-mass galaxies at $z \sim 1$, and extend them to lower $(z \sim 0.4)$ and higher $(z \sim 1.43)$ redshifts.

We performed a numerical integration of the number density of the OTELO galaxies and data in the literature. No evolution for low-mass galaxies was found.
Taking into account all of these effects, the resulting picture is a constant number density of galaxies, regardless of their masses, up to redshift $z \sim 1.43$. At the same time, there is a gradual change of the relative importance of the star-forming processes, from high-mass galaxies to low-mass galaxies with decreasing redshift. The contribution of low-mass galaxies is constant with redshift up to $z \sim 1.43$, while the contribution of high-mass galaxies increases with redshift. This may indicate the presence of the downsizing effect, as described by Cowie et al. (1996).

We can conclude that the low-mass galaxies are a valid baseline comparator of the star formation activity independently of the redshift window and the mass range considered. This allows a new approach to the study of the evolution of the SFR of intermediate to high-mass galaxies along the cosmic times.

The authors thank the anonymous referee for their feedback and constructive suggestions, which have contributed to significantly improve the manuscript. This work was supported by the Spanish Ministry of Economy and Competitiveness (MINECO) under the grants AYA2014-58861-C3-1-P, AYA2014 - 58861 - C3 - 2 - P, AYA2014 - 58861 - C3 - 3 - P, AYA2013 - 46724 - P, AYA2017 - 88007 - C3 - 1 - P, AYA2017 - 88007 - C3 - 2, MDM - 2017 - 0737 (Unidad de Excelencia María de Maeztu, CAB). A.P.G. acknowledge support from ESA through the Faculty of the European Space Astronomy Centre (ESAC)_Funding reference ESAC_549/ 2019. B.C. wishes to thank Carlota Leal Álvarez by her support during the development of this paper. E.J.A. acknowledges funding from the State Agency for Research of the Spanish MCIU through the "Center of Excellence Severo Ochoa" award to the Instituto de Astrofísica de Andalucía (SEV-2017-0709) and from grant PGC2018-095049-B-C21. Based on observations made with the Gran Telescopio Canarias (GTC), installed in the Spanish Observatorio del Roque de los Muchachos of the Instituto de Astrofísica de Canarias, in the island of La Palma.

This study makes use of data from AEGIS, a multiwavelength sky survey conducted with the Chandra, GALEX, Hubble, Keck, CFHT, MMT, Subaru, Palomar, Spitzer, VLA, and other telescopes and supported in part by the NSF, NASA, and the STFC.

Based on observations obtained with MegaPrime/MegaCam, a joint project of CFHT and CEA/IRFU, at the CanadaFrance-Hawaii Telescope (CFHT) which is operated by the National Research Council (NRC) of Canada, the Institut National des Science de l'Univers of the Centre National de la Recherche Scientifique (CNRS) of France, and the University of Hawaii. This work is based in part on data products produced at Terapix available at the Canadian Astronomy Data Centre as part of the Canada-France-Hawaii Telescope Legacy Survey, a collaborative project of NRC and CNRS.

Based on observations obtained with WIRCam, a joint project of CFHT, Taiwan, Korea, Canada, France, at the Canada-France-Hawaii Telescope (CFHT), which is operated by the National Research Council (NRC) of Canada, the Institute National des Sciences de l'Univers of the Centre National de la Recherche Scientifique of France, and the University of Hawaii. This work is based in part on data products produced at TERAPIX, the WIRDS (WIRcam Deep Survey) consortium, and the Canadian Astronomy Data Centre. 
This research was supported by a grant from the Agence Nationale de la Recherche ANR-07-BLAN-0228.

\section{ORCID iDs}

Bernabé Cedrés ํㅣ https://orcid.org/0000-0002-7382-6407

Jesús Gallego (1) https://orcid.org/0000-0003-1439-7697

Jakub Nadolny (i) https://orcid.org/0000-0003-1440-9061

Maritza A. Lara-López (i) https://orcid.org/0000-0001-

7327-3489

\section{References}

Bongiovanni, Á., Ramón-Pérez, M., Pérez García, A. M., et al. 2019, A\&A, 631, A9

Bongiovanni, Á., Ramón-Pérez, M., Pérez García, A. M., et al. 2020, A\&A, 635, A35

Brinchmann, J., Charlot, S., White, S. D. M., et al. 2004, MNRAS, 351, 1151

Calzetti, D., Armus, L., Bohlin, R. C., et al. 2000, ApJ, 533, 682

Cedrés, B., Bongiovanni, Á., Cerviño, M., et al. 2021, A\&A, 649, A73

Cerviño, M., Bongiovanni, A., \& Hidalgo, S. 2016, A\&A, 589, A108

Coleman, G. D., Wu, C. C., \& Weedman, D. W. 1980, ApJS, 43, 393

Cowie, L. L., Songaila, A., Hu, E. M., \& Cohen, J. G. 1996, AJ, 112, 839

Davies, G. T., Gilbank, D. G., Glazebrook, K., et al. 2009, MNRAS, 395, L76

Drake, A. B., Simpson, C., Collins, C. A., et al. 2013, MNRAS, 433, 796

Gilbank, D. G., Baldry, I. K., Balogh, M. L., Glazebrook, K., \& Bower, R. G. 2010, MNRAS, 405, 2594

Gilbank, D. G., Bower, R. G., Glazebrook, K., et al. 2011, MNRAS, 414, 304

González, V., Bouwens, R., Illingworth, G., et al. 2014, ApJ, 781, 34

Harish, S., Coughlin, A., Rhoads, J. E., et al. 2020, ApJ, 892, 30
Hayashi, M., Sobral, D., Best, P. N., Smail, I., \& Kodama, T. 2013, MNRAS, 430, 1042

Henry, A., Scarlata, C., Domínguez, A., et al. 2013, ApJL, 776, L27

Ilbert, O., Arnouts, S., McCracken, H. J., et al. 2006, A\&A, 457, 841

Kennicutt, Robert C., J. 1998, ARA\&A, 36, 189

Kewley, L. J., Geller, M. J., \& Jansen, R. A. 2004, AJ, 127, 2002

Khostovan, A. A., Sobral, D., Mobasher, B., et al. 2015, MNRAS, 452, 3948

Kinney, A. L., Calzetti, D., Bohlin, R. C., et al. 1996, ApJ, 467, 38

Kroupa, P. 2001, MNRAS, 322, 231

López-Sanjuan, C., Díaz-García, L. A., Cenarro, A. J., et al. 2019, A\&A, 622, A51

Ly, C., Malkan, M. A., Kashikawa, N., et al. 2007, ApJ, 657, 738

Mobasher, B., Dahlen, T., Hopkins, A., et al. 2009, ApJ, 690, 1074

Morioka, T., Nakajima, A., Taniguchi, Y., et al. 2008, PASJ, 60, 1219

Nadolny, J., Lara-López, M. A., Cerviño, M., et al. 2020, A\&A, 636, A84

Navarro Martínez, R., María Pérez-García, A., Pérez-Martínez, R., et al. 2021, arXiv:2105.13374

Ramón-Pérez, M., Bongiovanni, Á., Pérez García, A. M., et al. 2019, A\&A, 631, A10

Ramraj, R., Gilbank, D. G., Blyth, S.-L., et al. 2017, MNRAS, 466, 3143

Renzini, A., \& Peng, Y.-j 2015, ApJL, 801, L29

Rodríguez-Muñoz, L., Gallego, J., Pacifici, C., et al. 2015, ApJ, 799, 36

Schaye, J., Crain, R. A., Bower, R. G., et al. 2015, MNRAS, 446, 521

Schechter, P. 1976, ApJ, 203, 297

Sobral, D., Best, P. N., Smail, I., et al. 2011, MNRAS, 411, 675

Sobral, D., Smail, I., Best, P. N., et al. 2013, MNRAS, 428, 1128

Stroe, A., \& Sobral, D. 2015, MNRAS, 453, 242

Vogelsberger, M., Genel, S., Springel, V., et al. 2014, MNRAS, 444, 1518

Weisz, D. R., Dalcanton, J. J., Williams, B. F., et al. 2011, ApJ, 739, 5

Whitaker, K. E., Franx, M., Leja, J., et al. 2014, ApJ, 795, 104 